\title{
1 Serological screening suggests single SARS-CoV-2 spillover events to cattle
}

3 Authors: Kerstin Wernike, Jens Böttcher, Silke Amelung, Kerstin Albrecht, Tanja Gärtner,

$4 \quad$ Karsten Donat, Martin Beer

5

\section{Affiliations:}

7 Friedrich-Loeffler-Institut, Greifswald - Insel Riems, Germany (K. Wernike, M. Beer)

8 Bavarian Animal Health Service, Poing, Germany (J. Böttcher)

9 LUFA Nord-West, Oldenburg, Germany (S. Amelung)

10 State Institute for Consumer Protection of Saxony-Anhalt, Stendal, Germany (K. Albrecht)

11 Thuringian Animal Diseases Fund, Animal Health Service, Jena, Germany (T. Gärtner, K.

12 Donat) 


\section{Abstract}

Widespread human SARS-CoV-2 infections pose a constant risk for virus transmission to

15 animals. Here, we serologically investigated 1000 cattle samples collected in late 2021 in

16 Germany. Eleven sera tested antibody-positive, indicating that cattle may be occasionally

17 infected by contact to SARS-CoV-2-positive keepers, but there is no indication of further

18 spreading.

20 Keywords: SARS-CoV-2, COVID-19, animal, reservoir, cattle, ruminants, livestock, serology,

21 epidemiology 


\section{Text}

Since its first detection at the end of 2019, the betacoronavirus SARS-CoV-2 is keeping the world in suspense. This novel virus, which induces coronavirus disease 2019 (COVID-19) in humans, very rapidly spread around the world, thereby causing a massive global pandemic that resulted in more than five millions of deaths in less than two years of virus circulation (1). Since

27 the beginning of the pandemic, the role of livestock and wildlife species at the human-animal

28 interface was discussed. A special focus was placed on the identification of susceptible species and potential intermediate or reservoir hosts. Under experimental conditions, various animal species could be infected with SARS-CoV-2, among them non-human primates, felines, canines, mustelids, white-tailed deer and several Cricetidae species, while e.g. poultry or swine are not susceptible (2). For domestic ruminants such as cattle, sheep or goat a very low susceptibility was demonstrated following experimental inoculation, as only a small proportion of animals could be infected without animal to animal transmission (3-5). Furthermore, 26 cattle exposed in the field to SARS-CoV-2 via contact to their infected keepers tested negative by RT-PCR (6). However, given the very short time frame of only one to two days at which cattle test RT-PCR positive after experimental infection (3,7), serological screenings could be more beneficial to identify previously infected animals, in order to estimate the rate of spill-over infections in the field.

Here, 1000 available samples of cattle kept in 83 holdings located in four German federal states (Bavaria, Lower Saxony, Saxony-Anhalt and Thuringia) were analyzed. The sampling

42 dates were autumn 2021 and early winter 2021/22 when a massive wave of infections in the

43 human population driven by the Delta variant of concern (VOC) occurred. Two to 20 randomly

44 selected serum or plasma samples were analyzed per holding. Farm 31 was sampled twice, in 
45 between the animal owner was quarantined. Whether this quarantine was due to contact to an

46 infected person or whether the owner himself tested SARS-CoV-2 positive is not known to the

47 authors. All bovine samples were tested by an RBD-based multispecies ELISA performed as

48 described previously (8). During the initial test validation and during an experimental SARS-

49 CoV-2 infection study in cattle, it could be shown that the ELISA does not cross-react with the

50 bovine coronavirus (BCoV) (3,8). Here, additional 100 cattle control samples randomly collected

51 across Germany in 2016 were investigated and all of them tested negative.

Of the animals sampled in 2021, 11 cattle from nine farms tested positive by the RBD-

(Figure 1). All but one (farm 8) positive ELISA results could be confirmed by an indirect between $1 / 8$ and $1 / 512$, where the highest titer was measured in the seropositive animal from reacted positive in the RBD-ELISA were additionally tested by a surrogate virus neutralization

61 Netherlands). This test allows for the detection of neutralizing antibodies by mimicking the

62 interaction between SARS-CoV-2 and the host cell's membrane receptor protein ACE2. It was

63 reported to be highly specific but only moderately sensitive for animal samples, since it does not

64 detect low antibody titers (9). Four cattle samples scored also positive by the sVNT (farms 11,

6531,47 and 74; Table 1). 
seroconvert. However, in keeping with experimental infection studies (3), intraspecies transmission seems likewise not to occur in the field. Nevertheless, cattle farms should be included in future monitoring programs, especially as another coronavirus, i.e. BCoV, is highly

71 prevalent in the cattle population and a $\mathrm{BCoV}$ infection did not prevent a SARS-CoV-2 infection

72 in a previous study (3). Furthermore, we do not know the susceptibility of animal hosts for the 73 new VOC Omicron. between both viruses, a phenomenon well-described for other coronaviruses (10). Although, the emergence is highly unlikely due to the low susceptibility of cattle for SARS-CoV-2, a

77 conceivable chimera between SARS-CoV-2 and BCoV could represent an additionally threat.

78 Hence, also ruminants should be included in outbreak investigations and regular screenings

79 should be performed to exclude any spread of new variants in the livestock population.

\section{Acknowledgments}

We thank Bianka Hillmann and Mareen Lange for excellent technical assistance. The

83 study was supported by intramural funding of the German Federal Ministry of Food and

84 Agriculture provided to the Friedrich-Loeffler-Institut.

\section{Ethical Statement}




\section{References}

90 1. Dong E, Du H, Gardner L. An interactive web-based dashboard to track COVID-19 in

91 real time. Lancet Infect Dis. 2020 May;20(5):533-4.

92 2. Michelitsch A, Wernike K, Ulrich L, Mettenleiter TC, Beer M. SARS-CoV-2 in animals:

93 From potential hosts to animal models. Adv Virus Res. 2021;110:59-102.

94 3. Ulrich L, Wernike K, Hoffmann D, Mettenleiter TC, Beer M. Experimental infection of

95 cattle with SARS-CoV-2. Emerg Infect Dis. 2020 Dec;26(12):2979-81.

96 4. Gaudreault NN, Cool K, Trujillo JD, Morozov I, Meekins DA, McDowell C, et al.

97 Susceptibility of sheep to experimental co-infection with the ancestral lineage of SARS-CoV-2

98 and its alpha variant. bioRxiv. 2021.

99 5. Bosco-Lauth AM, Walker A, Guilbert L, Porter S, Hartwig A, McVicker E, et al.

100 Susceptibility of livestock to SARS-CoV-2 infection. Emerg Microbes Infect. 2021

101 Dec;10(1):2199-201.

102 6. Cerino P, Buonerba C, Brambilla G, Atripaldi L, Tafuro M, Concilio DD, et al. No

103 detection of SARS-CoV-2 in animals exposed to infected keepers: results of a COVID-19

104 surveillance program. Future Sci OA. 2021 Aug;7(7):Fso711.

105 7. Falkenberg S, Buckley A, Laverack M, Martins M, Palmer MV, Lager K, et al.

106 Experimental inoculation of young calves with SARS-CoV-2. Viruses. 2021;13(3).

107 8. Wernike K, Aebischer A, Michelitsch A, Hoffmann D, Freuling C, Balkema-Buschmann

108 A, et al. Multi-species ELISA for the detection of antibodies against SARS-CoV-2 in animals.

109 Transbound Emerg Dis. 2021 Jul;68(4):1779-85. 
1109 9mbregts CWE, Verstrepen B, Langermans JAM, Böszörményi KP, Sikkema RS, de

111 Vries RD, et al. Evaluation of a multi-species SARS-CoV-2 surrogate virus neutralization test.

112 One Health. 2021 Dec;13:100313.

113 10. Forni D, Cagliani R, Clerici M, Sironi M. Molecular evolution of human coronavirus

114 genomes. Trends Microbiol. 2017 Jan;25(1):35-48.

116 Address for correspondence: Kerstin Wernike and Martin Beer, Institute of Diagnostic

117 Virology, Friedrich-Loeffler-Institut, Südufer 10, 17493 Greifswald - Insel Riems, Germany;

118 kerstin.wernike@fli.de, martin.beer@fli.de 
119 Table 1. Detailed information about the results of samples that tested positive by a multispecies

120 SARS-CoV-2 RBD-based ELISA. iIFA = indirect immunofluorescence assay, sVNT = surrogate

121 virus neutralization test (cPass SARS-CoV-2 Surrogate Virus Neutralization Test (sVNT) Kit,

122 GenScript, the Netherlands; cut-off $\geq 30 \%$ positive and $<30 \%$ negative)

\begin{tabular}{llll}
\hline Cattle farm/animal & RBD-ELISA & iIFA & sVNT \\
number & $($ corr. OD - status $)$ & (titer - status $)$ & (\% inhibition - status) \\
\hline $8 / 1$ & $\mathbf{0 . 3 5}$ - positive & $<1 / 8$ - negative & 6.1 - negative \\
$11 / 1$ & $\mathbf{0 . 7 0}$ - positive & $\mathbf{1 / 3 2}$ - positive & $\mathbf{3 6 . 4}$ - positive \\
$31 / 1$ & $\mathbf{1 . 0 0}$ - positive & $\mathbf{1 / 5 1 2}$ - positive & $\mathbf{5 7 . 8}$ - positive \\
$34 / 1$ & $\mathbf{0 . 5 0}$ - positive & $\mathbf{1 / 3 2}$ - positive & 11.7 - negative \\
$42 / 1$ & $\mathbf{0 . 6 5}$ - positive & $\mathbf{1 / 1 6}$ - positive & 5.5 - negative \\
$45 / 1$ & $\mathbf{0 . 6 7}$ - positive & $\mathbf{1 / 8}$ - positive & 10.6 - negative \\
$45 / 2$ & $\mathbf{0 . 3 3}$ - positive & $\mathbf{1 / 1 6}$ - positive & 9.0 - negative \\
$47 / 1$ & $\mathbf{0 . 4 8}$ - positive & $\mathbf{1 / 8}$ - positive & $\mathbf{3 7 . 1}$ - positive \\
$47 / 2$ & $\mathbf{0 . 6 7}$ - positive & $\mathbf{1 / 8}$ - positive & 0.6 - negative \\
$72 / 1$ & $\mathbf{0 . 5 2}$ - positive & $\mathbf{1 / 1 6}$ - positive & 4.7 - negative \\
$74 / 1$ & $\mathbf{0 . 7 6}$ - positive & $\mathbf{1 / 3 2}$ - positive & $\mathbf{5 4 . 2}$ - positive \\
& & &
\end{tabular}


Figure 1. Number of cattle per farm tested for antibodies against SARS-CoV-2. Samples that reacted negative in the RBD-based

ELISA are depicted in black and positive samples in red. Holding 31 was sampled twice (indicated as 31a and 31b), in between the animal owner was quarantined. negative in SARS-CoV-2 RBD-based ELISA

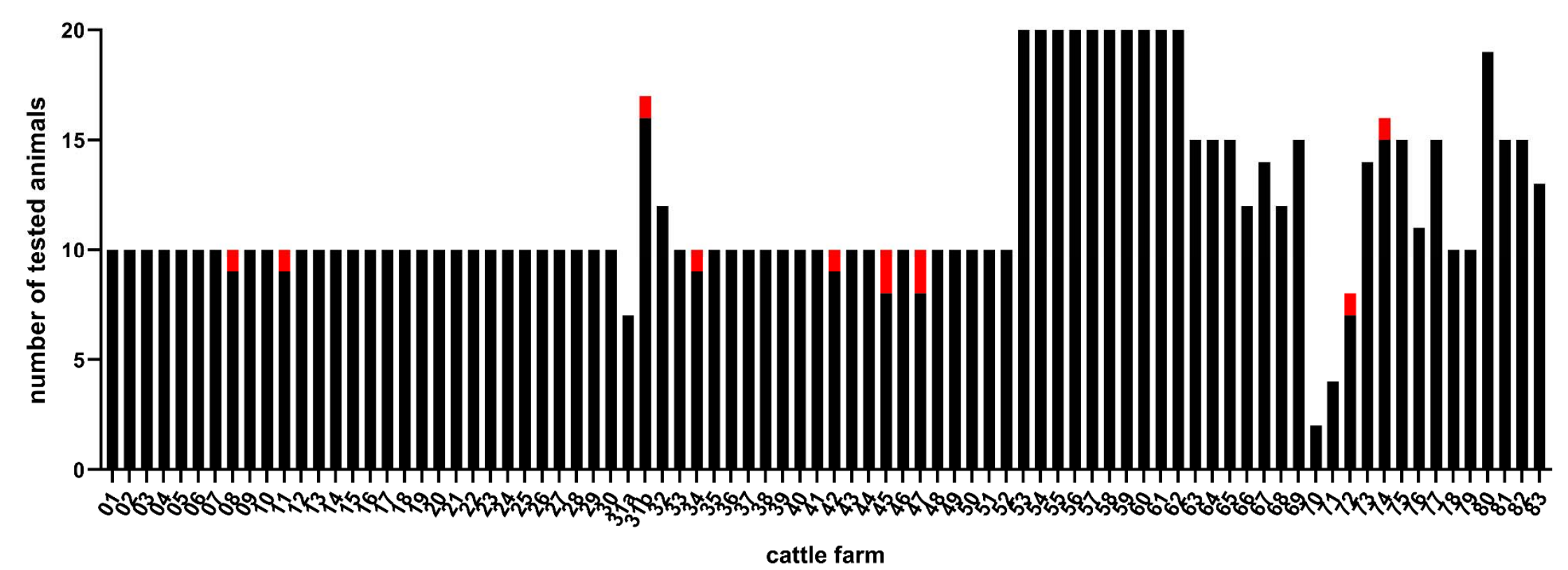

